\title{
Two Dimensional Spreading for Dispersive Channels
}

\author{
Joydeep Acharya, Roy D. Yates and Leonid Razoumov \\ Wireless Information Network Laboratory (WINLAB) \\ 73 Brett Rd, Piscataway, NJ 08854 \\ \{joy, ryates, leor\}@winlab.rutgers.edu
}

\begin{abstract}
Systems supporting broadband mobile services over wireless channels suffer from dispersion along time and frequency. Hence transmission by spreading information along both these dimensions leads to diversity gain in each dimension. Motivated by this principle, we propose a generalized two dimensional spreading scheme, in which a symbol is transmitted across several subcarriers with a total power constraint and along each subcarrier it is spread with CDMA codewords. The information theoretic bounds on capacity for this scheme are derived under different assumptions about the channel state information (CSI) available at the transmitter. These are perfect CSI, partial CSI characterized by one bit of channel information per subcarrier and no CSI. The receiver is assumed to have perfect CSI. The optimal codeword and power allocation strategies to achieve these bounds are derived for the single user point to point transmission. The solutions also apply to the cases of synchronous multi-user uplink transmission, or when the receiver schedules transmissions of multiple users. For asynchronous uplink communications, we show that the problem of optimal resource allocation is analytically intractable and instead suggest a heuristic solution which can be implemented in a distributed manner.
\end{abstract}

\section{INTRODUCTION}

Wireless channels are dispersive due to multipath fading and this can be effectively combated by transmission along multiple dimensions [2]. If the CSI is known at the transmitter, then availability of multiple dimensions, allows it to transmit along the eigenmodes of the channel, thus enhancing the achievable rates. Examples are waterfilling over parallel subcarriers in OFDM and transmit beamforming in MIMO. If the CSI is not known at transmitter, then signaling along multiple dimensions allows for diversity which improves error performance. In this paper we focus on multicarrier systems and hence by multiple dimensions we refer to time and frequency subcarriers. Systems like single carrier CDMA and MC-DS-CDMA system [12] achieve diversity by time domain spreading of data along a frequency subcarrier. In contrast MC-CDMA systems spread the same data along adjacent frequency subcarriers [16]. Recently multicarrier systems that use both time and frequency spreading have been proposed. Prominent examples are Multicarrier DS/CDMA [16] and VSF-OFCDM [7], which essentially spread a data symbol along time by conventional CDMA codewords and also along frequency by replicating the symbol along multiple subcarriers. This phenomenon is denoted by two dimensional spreading. Depending upon the channel conditions, the spread factors $\mathrm{SF}_{\text {Time }}$ (length of CDMA code in time) and $\mathrm{SF}_{\text {Freq }}$ (number of subcarriers on which the symbol is replicated) are adaptively controlled. Most of the available literature about these schemes, pertain to simulation studies [7], [16]. In this work we aim to study two dimensional spreading from an information-theoretic framework. We propose a generalized two-dimensional spreading model in which a symbol is transmitted across several subcarriers with a total power constraint and along each subcarrier it is spread with CDMA codewords. Note that all the aforementioned spreading models are special cases of this scheme. In the presence of channel state information at the transmitter, multiple subcarriers are used to transmit along the optimal direction and in its absence they are used for diversity. We study optimal power allocation policies along subcarriers and achievable rates using this twodimensional spreading scheme. Accordingly the rest of the paper is organized as follows: in Section II the channel model is described. In Section III, the two dimensional spreading system and the notations are described. Section IV discusses the single user case, the results of which are applicable to synchronous multiuser communications. Section V similarly analyzes the asynchronous multiuser case and we then conclude in Section VI.

\section{The Channel Model}

A block fading parallel channel model is assumed, i.e the subcarriers have i.i.d. channel gains, and along a subcarrier the value of its channel gain stays constant for the duration of the CDMA codeword. Such a model is an useful abstraction for a wide class of practical channels including the next generation cellular data channels [7]. Also recent advances in orthogonal basis function designs for wireless channels [6], promise to make even the most general doubly-dispersive channel assume a block fading baseband representation.

\section{System Model AND Notations}

In the two-dimensional spreading scheme, each symbol is replicated over $n_{f}$ subcarriers, with certain power and along each subcarrier they are spread by a unit norm CDMA code of length $n_{t}$ chips. There is a total power constraint on the powers allocated in the $n_{f}$ subcarriers.

\section{A. Notation}

Throughout this paper we use uppercase boldface letters to denote matrices, lowercase boldface letters to denote 


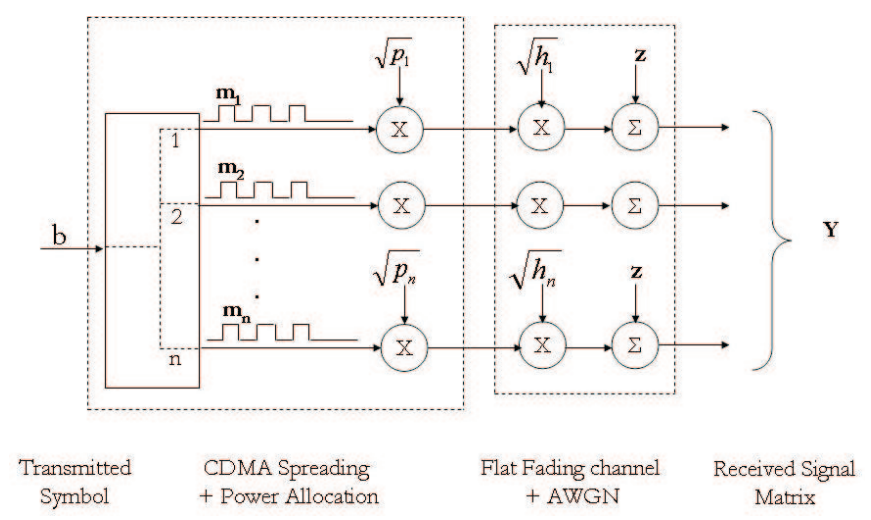

Fig. 1. Transmitter Block Diagram

vectors and lowercase letters to denote scalars. In particular we adopt the following notations: the subscript $i$ denotes parameters of user $i$, the subscript $j$ denotes the index of the $j^{\text {th }}$ subcarrier and the subscript $k$ denotes the time slot index along a subcarrier. Each time slot is occupied by a CDMA chip.

The symbol $b_{i}$ denotes the $i^{\text {th }}$ user's data symbol. We use $\mathbf{M}_{i}=\left\{m_{i j k}\right\}, 1 \leq j \leq n_{f}, 1 \leq k \leq n_{t}$ to denote the $n_{f} \times n_{t}$ spreading code matrix for the $i^{t h}$ user and $\mathbf{m}_{i j}$ to denote the $i^{\text {th }}$ user's spreading code along the $j^{\text {th }}$ subcarrier. The matrices $\mathbf{P}_{i}$ and $\mathbf{H}_{i}$ are the $n_{f} \times n_{f}$ diagonal matrices of powers and channel gains respectively for user $i$, i.e. $\mathbf{P}_{i}=\operatorname{diag}\left\{\mathbf{p}_{i}\right\}$, where $\mathbf{p}_{i}=\left[p_{i 1}, p_{i 2}, \cdots, p_{i n_{f}}\right]$ and $\mathbf{H}_{i}=\operatorname{diag}\left\{\mathbf{h}_{i}\right\}$, where $\mathbf{h}_{i}=\left[h_{i 1}, h_{i 2}, \cdots, h_{i n_{f}}\right]$. If $\mathbf{x}=\left[x_{1}, \cdots, x_{n}\right]$, then $\mathbf{x}^{\frac{1}{2}}$ denotes the vector $\left[\sqrt{x_{1}}, \cdots, \sqrt{x_{n}}\right]$ and a similar notation is valid for diagonal matrices.

\section{Single USER/Multiuser SynChronous TRANSMISSION}

We consider the case of a single transmitter using two dimensional spreading to communicate to a receiver. Though a simplified system, single user transmission gives important analytical insights about the nature of two dimensional spreading. Also this models two important multi-user scenarios - firstly the multiuser MAC channel, when each transmitter has a set of orthogonal CDMA codes and synchronous reception at the receiver is assumed. The second scenario occurs when the central receiver schedules the transmission of various users and hence at a time only one user is active.

For the single user analysis, we drop the user index $i$ for notational simplicity. The received signal matrix $\mathbf{Y}$ is given by,

$$
\mathbf{Y}=\mathbf{H}^{\frac{1}{2}} \mathbf{P}^{\frac{1}{2}} \mathbf{M} b+\mathbf{Z} .
$$

Given a particular fading distribution, we try to evaluate the optimal power allocation and codeword assignment policies to maximize the achievable rates.

\section{A. Perfect CSI at Transmitter}

In this section we assume that transmitter and receiver are both equipped with instantaneous values of CSI, for a block of $n_{f} n_{t}$ transmitted symbols. If the channel realization changes from block to block, the ergodic capacity is defined as the average of the achievable rates for each channel realization [13]. For the single subcarrier case with no spreading, Caire et al [3] gave an expression for ergodic capacity and a fixed rate coding scheme to achieve this capacity. Assuming that the CDMA codes along each subcarrier are orthonormal, the ergodic capacity maximization problem can be formulated along the lines of [3] as,

$$
\begin{aligned}
\mathrm{C}_{\mathrm{PCSI}} & =\max _{\mathbf{p}(\mathbf{h})} \int \cdots \int R(\mathbf{h}) f(\mathbf{h}) d \mathbf{h} \\
& \text { s.t. } \sum_{j=1}^{n_{f}} \int \cdots \int p_{j}(\mathbf{h}) f(\mathbf{h}) d \mathbf{h}=\bar{P}, \quad p_{j}(\mathbf{h}) \geq 0,
\end{aligned}
$$

where

$$
R(\mathbf{h})=\frac{1}{2} \log \left(1+\frac{1}{\sigma^{2}} \sum_{j=1}^{n_{f}} h_{j} p_{j}(\mathbf{h})\right),
$$

is termed as the maximum mutual information (MMI) for a given CSI $\mathbf{h}$. For every block, when the CSI is revealed to the transmitter and receiver, the optimal solution to (2) is to transmit only in that subcarrier which has the highest channel gain (henceforth referred to as the best subcarrier), i.e. in subcarrier $i^{*}=\arg \max h_{i}$. We denote the random variable $h_{i^{*}}$ by $h_{n}^{*}$, where $n$ is the number of random variables over which the maximization operation has been performed. In this case $n=n_{f}$, the number of subcarriers. The power, $p\left(h_{n}^{*}\right)$ allocated to the best subcarrier is obtained by waterfilling over the distribution of $h_{n}^{*}$, as

$$
p\left(h_{n}^{*}\right)=\left(\frac{1}{\lambda}-\frac{\sigma^{2}}{h_{n}^{*}}\right)^{+},
$$

where $\lambda$, is found by substituting for $p\left(h_{n}^{*}\right)$ in Equation (3), as

$$
\int\left(\frac{1}{\lambda}-\frac{\sigma^{2}}{\gamma}\right)^{+} f_{h_{n}^{*}}(\gamma) d \gamma=\bar{P}
$$

The water-filling level is given by $1 / \lambda$. Similar results were reported in [5], where the problem was to maximize sum capacity for multiuser scalar MAC channel. The solution was to let only the user with the highest channel gain transmit. The transmitted powers were obtained by waterfilling over the distribution of the channel gain of this user. We obtain similar results here for a different physical situation, as both have similar signal space models.

The optimization of codewords amounted to selecting any set of unit norm, orthogonal codewords. The CDMA spreading along subcarriers had no effect on the ergodic 


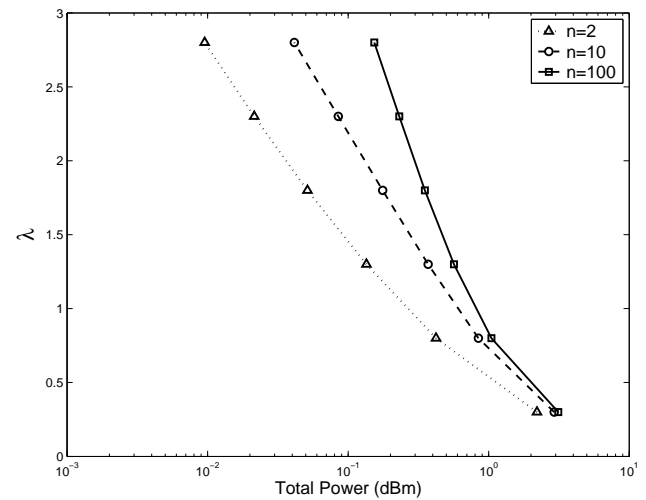

Fig. 2. Rayleigh fading: water-filling parameter $\lambda$ vs total power for $n$ subcarriers: $n=2,10,100$

capacity as given by Equations (2) and (4). This is consistent with the observation that CDMA doesn't increase capacity for single user transmission [14]. Also in the multi-user case, CDMA capacity is upper bounded by the capacity of the unspread transmission [15].

1) Numerical Results for Rayleigh Fading: Each subcarrier undergoes independent Rayleigh fading so the distribution of the $h_{i}$ are exponential. The mean of the fading coefficient is assumed to be unity. Equation (6) relates $\lambda$ to $\bar{P}$ and has to be solved numerically. Figure (2) gives the results of this numerical computation. The following observations are readily made:

- For same $n$, a decrease in total power $\bar{P}$, leads to an increase in $\lambda$, which increases the threshold, $\lambda \sigma^{2}$ above which transmission takes place. This implies that when $\bar{P}$ is low, the transmitter has to wait for a really good channel to transmit. This suggests that the fraction of time the transmitter is turned on is less. Similar results are reported in [5].

- For the same total power, $\bar{P}$, increasing $n$ increases $\lambda$, which raises the threshold. This is because having more subcarriers raises the probability of encountering a higher channel gain $h_{n}^{*}$.

2) Numerical Results for Uniform Fading: We first consider that the uniform fading channel [3] i.e. the $\mathbf{h}_{i}$ are uniformly distributed in $[0,1]$. Figure 3 plots the variations in the MMI sequence for SNR $=1 \mathrm{~dB}$, by increasing $n \equiv n_{f}$, number of subcarriers . The term SNR is used to denote $\bar{P} / \sigma^{2}$, where $\bar{P}$ is defined in Equation (3). We make the following observations:

- The MMI (and hence the ergodic capacity) increases for more subcarriers as the higher values of $h_{n}^{*}$ becomes more probable.

- The fluctuations in MMI decrease for more subcarriers, as variance of $h_{n}^{*}$ decreases.

- In the regime of large $n$, the channel gain tends to unity with probability one and hence the MMI tends to a constant.

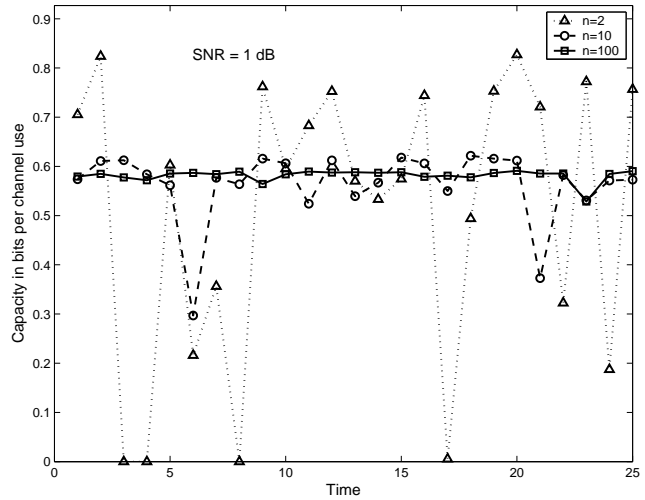

Fig. 3. Uniform fading: MMI variations with time for $S N R=1 d B$

Hence we conclude that frequency spreading, led to higher achievable rates as the transmitter had the freedom to transmit along better channels.

\section{B. Imperfect CSI at Transmitter}

In most practical systems CSI at transmitter is due to receiver feedback, which can be erroneous [2]. In this section, we thus assume that perfect CSI is not available at the transmitter and investigate its effects upon ergodic channel capacity. The receiver is still assumed to possess perfect CSI. Quantitatively, let the true channel state be $\mathbf{h}$ and the CSI at the transmitter be $\mathbf{u}$. The optimal solution depends on the correlation between $\mathbf{u}$ and $\mathbf{h}$ as characterized by the conditional density $f(\mathbf{h} \mid \mathbf{u})$ [3]. The ergodic capacity optimization problem can be written as

$$
\begin{aligned}
& \max _{p(\mathbf{u})} \int \cdots \int R(\mathbf{h}, \mathbf{u}) f(\mathbf{h} \mid \mathbf{u}) d \mathbf{h} \\
\text { s.t. } & \sum_{j=1}^{n_{f}} \int \cdots \int p_{j}(\mathbf{u}) f(\mathbf{u}) d \mathbf{u}=\bar{P}, \quad p_{j}(\mathbf{u}) \geq 0,
\end{aligned}
$$

where $R(\mathbf{h}, \mathbf{u})$, the MMI is given by,

$$
R(\mathbf{h}, \mathbf{u})=\frac{1}{2} \log \left(1+\frac{1}{\sigma^{2}} \sum_{j=1}^{n_{f}} h_{j} p_{j}(\mathbf{u})\right) .
$$

Note that the transmit powers are a function of $\mathbf{u}$. The Lagrangian for the optimization problem is,

$$
\begin{aligned}
J & =\int \cdots \int R(\mathbf{h}, \mathbf{u}) f(\mathbf{h} \mid \mathbf{u}) f(\mathbf{u}) d \mathbf{h} d \mathbf{u} \\
& -\quad \lambda \sum_{j=1}^{n_{f}} \int \cdots \int p_{j}(\mathbf{u}) f(\mathbf{u}) d \mathbf{u} .
\end{aligned}
$$

Differentiating with respect to $p_{j}(\mathbf{u})$ we obtain

$$
\frac{\partial J}{\partial p_{j}(\mathbf{u})}=s_{j}(\mathbf{u})-\lambda
$$

where

$$
s_{j}(\mathbf{u})=\frac{1}{2} \int \cdots \int\left(\frac{h_{j}}{\sigma^{2}+\sum_{k=1}^{n_{f}} h_{k} p_{k}(\mathbf{u})}\right) f(\mathbf{h} \mid \mathbf{u}) d \mathbf{h} .
$$


The Kuhn-Tucker conditions state $\partial J / \partial p_{j}(\mathbf{u})=0$ if $p_{j}(\mathbf{u}) \neq 0$ and $\partial J / \partial p_{j}(\mathbf{u}) \leq 0$ if $p_{j}(\mathbf{u})=0$ for all $j$.

The maximum value of $s_{j}(\mathbf{u})$ occur when $p_{j}(\mathbf{u})=0$ for all $j$. This value is

$$
\frac{1}{2 \sigma^{2}} \int \cdots \int h_{j} f(\mathbf{h} \mid \mathbf{u}) d \mathbf{h}=\frac{1}{2 \sigma^{2}} E\left[h_{j} \mid u_{j}\right] .
$$

Lets denote this maximum value by $s_{j}^{*}\left(u_{j}\right)$. Note that the conditional fading distribution of $h_{j}$ given $u_{j}$ is i.i.d. in subcarriers $j=p$ and $j=q$ if $u_{p}=u_{q}=u$. Hence $s_{p}^{*}\left(u_{p}\right)=s_{q}^{*}\left(u_{q}\right)$. Transmission takes place in the $j^{t h}$ subcarrier only if $s_{j}^{*}\left(u_{j}\right)>\lambda$. Hence $s_{j}^{*}\left(u_{j}\right)$ gives the threshold for transmission in the $j^{\text {th }}$ subcarrier. Note that $\lambda$ depends on $\bar{P}$, the total average power and thus these threshold inequalities can also be expressed in terms of $\bar{P}$. Let us apply these results for an example system in which $u_{k}$ is the one bit quantized information about the channel state at subcarrier $k$ [3], i.e.

$$
u_{k}= \begin{cases}0 & h_{k}<h_{T h}, \\ 1 & h_{k} \geq h_{T h} .\end{cases}
$$

Two cases arise from this feedback structure:

1) At least one subcarrier receives $u=1$ : Let $m$ subcarriers receive unity feedback, where $1 \leq m \leq n_{f}$. Since the conditional densities $f\left(h_{j} \mid u_{j}\right)$ are same for all these $m$ subcarriers, the optimal solution is a symmetric policy that allocates equal power to all these $m$ subcarriers. Let us denote this power by $\pi(m)$. It can be further shown that no power should be transmitted in the remaining $n_{f}-$ $m$ subcarriers [1]. Without loss of generality we assume that $u_{j}=1$ for $1 \leq j \leq m$ and define $\mathbf{u}_{m}=[\underbrace{1, \cdots, 1}_{m}]$ and $\mathbf{h}_{m}=\left[h_{1}, \cdots, h_{m}\right]$. The value of $\pi(m)$ is determined by solving any one of the $m$ equations, $\partial J / \partial p_{j}\left(\mathbf{u}_{m}\right)=0$, s.t. $u_{j}=1$. Solving the first equation we obtain,

$\frac{1}{2} \int \cdots \int\left(\frac{h_{1}}{\sigma^{2}+\pi(m) \sum_{k=1}^{m} h_{k}}\right) f\left(\mathbf{h}_{m} \mid \mathbf{u}_{m}\right) d \mathbf{h}_{m}=\lambda$.

From Equation (13) the value $\pi(m)$ is non-zero if

$$
s_{j}^{*}\left(u_{j}=1\right) \geq \lambda,
$$

where $s_{j}^{*}\left(u_{j}=1\right)$ is same for all $\mathrm{j}, 1 \leq j \leq m$.

2) All $n_{f}$ subcarriers receive $u=0$ : The solution, obtained along lines of the previous case, is to let all users transmit at the same power $\pi(0)$ which is given by,

$$
\frac{1}{2} \int \cdots \int\left(\frac{h_{1}}{\sigma^{2}+\pi(0) \sum_{k=1}^{n_{f}} h_{k}}\right) f(\mathbf{h} \mid \mathbf{u}) d \mathbf{h}=\lambda,
$$

where all components of $\mathbf{u}$ are 0 . The power, $\pi(0)$ is nonzero if

$$
s_{j}^{*}\left(u_{j}=0\right) \geq \lambda .
$$

It can be shown that the threshold $s_{j}^{*}(u=1)$ is higher than $s_{j}^{*}(u=0)$ as in the former case all subcarriers have better values of conditional channel gains.

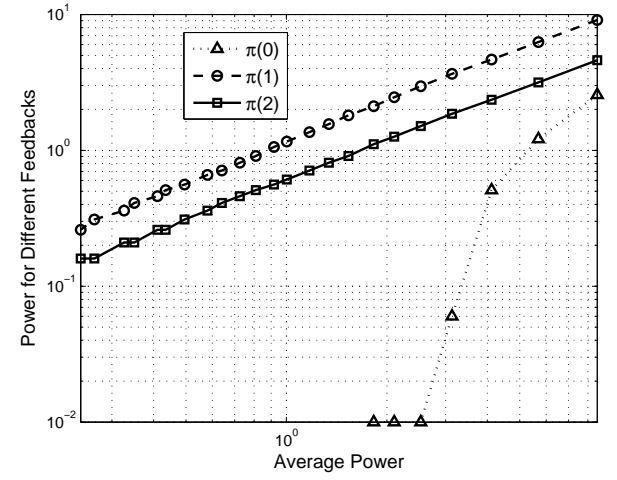

Fig. 4. Plot of power allocated for different feedbacks vs average power

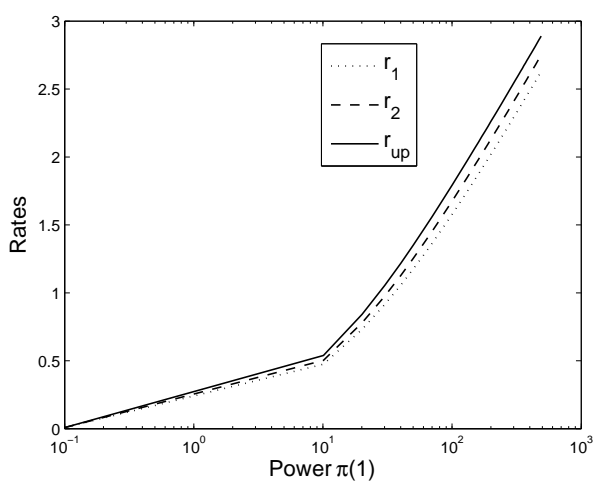

Fig. 5. Plot of average rates vs Power $\pi(1)$ assuming $\pi(1)=2 \pi(2)$

Note that similar results were reported in [8], for the related problem of multi-user, single carrier transmission.

Let us consider $n_{f}=2$ and plot the powers $\pi(0), \pi(1)$ and $\pi(2)$ vs the total power $\bar{P}$, in Figure 4 . We consider Rayleigh fading and the threshold $h_{T h}$ of Equation (14) is 0.5 . It is seen that for low $\bar{P}$, no power is allocated to the all zero feedback case. Another observation is that $\pi(1) \sim$ $2 \pi(2)$. An intuitive explanation is that the same power gets allocated to one subcarrier, as $\pi(1)$ and is divided into two subcarriers as $\pi(2)$ in each. Assuming $\pi(1)=2 \pi(2)$, we plot the average achievable rate $r_{2}$ corresponding to power $\pi(2)$ and rate $r_{1}$ corresponding to power $\pi(1)$, for $h_{T h}=0.1$ in Figure 5. The upper bound to these rates, $r_{u p}$ is also plotted. It is seen that there $r_{2}$ is slightly higher than $r_{1}$, which can be attributed to the diversity gain in transmitting in two subcarriers.

We now consider the case when the transmitter has no CSI. It can be shown that [10] the capacity maximizing policy is constant power allocation across all subcarriers i.e. $p_{j}(\mathbf{u})=\bar{P} / n_{f}$. Thus the upper bound to achievable 


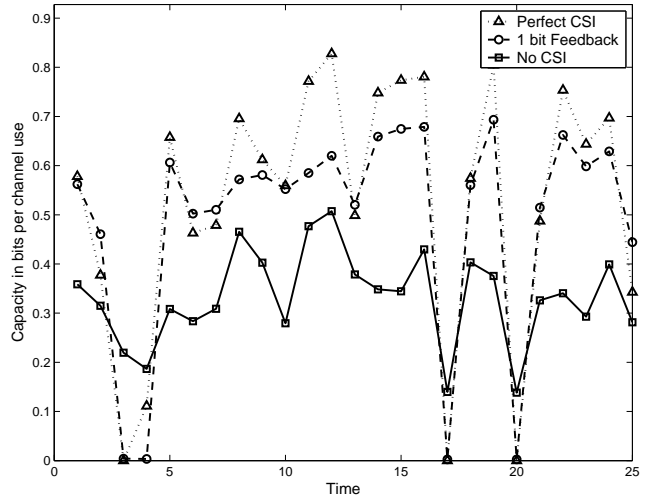

Fig. 6. MMI for different types of CSI

rates is,

$$
\begin{aligned}
C_{\text {NCSI }} & =E_{\mathbf{h}}\left[\frac{1}{2} \log \left(1+\frac{1}{\sigma^{2}} \sum_{j=1}^{n_{f}} h_{j}\left(\frac{\bar{P}}{n_{f}}\right)\right)\right] \\
& \leq \frac{1}{2} \log \left(1+\frac{\overline{h P}}{\sigma^{2}}\right),
\end{aligned}
$$

by Jensen's Inequality. Note that the MMI expression for $p_{j}(\mathbf{u})=\bar{P} / n_{f}$, is

$$
R_{\mathrm{NCSI}}=\frac{1}{2} \log \left(1+\frac{1}{\sigma^{2}} \frac{1}{n_{f}} \sum_{j=1}^{n_{f}} h_{j} \bar{P}\right) .
$$

We observe that as $n_{f} \longrightarrow \infty$,

$$
R_{\mathrm{NCSI}} \longrightarrow \frac{1}{2} \log \left(1+\frac{\bar{h} \bar{P}}{\sigma^{2}}\right),
$$

by the law of large numbers. Note that for a single subcarrier the corresponding expression for MMI with uniform power allocation is $\log \left(1+h \bar{P} / \sigma^{2}\right) / 2$, and there is no way to actually achieve this bound. This can be looked upon as the diversity advantage offered by multiple subcarriers.

Note that in the two cases of imperfect CSI considered, two-dimensional spreading was useful as it lead to diversity gains. Finally Figure 6 plots the MMI variations with time for all the three types of CSI. We observe that for $n_{f}=2$, the achievable rates with 1 bit per subcarrier feedback is close to the perfect CSI case. This suggests that the 1 bit per subcarrier scheme is a good practical scheme for transmitter feedback. In some time instants the MMI achieved for the perfect CSI is lower, because the perfect CSI solution waits for the occurance of a good channel state to allocate power.

\section{Multiuser ASynchronous Transmission}

In this section, we consider asynchronous transmissions, due to which users do not orthogonalize at the receiver. Such a situation might also arise if there are more users than orthogonal CDMA codewords. We assume that there are $N$ users in the system. The multi-user transmission given by,

$$
\mathbf{Y}=\sum_{i=1}^{N} \mathbf{H}_{i}^{\frac{1}{2}} \mathbf{P}_{i}^{\frac{1}{2}} \mathbf{M}_{i} b_{i}+\mathbf{Z} .
$$

The problem of maximizing the ergodic sum capacity $E[I(\mathbf{Y} ; \mathbf{b})]$, where $\mathbf{b}=\left[b_{1}, \cdots, b_{N}\right]$ over choice of power and spreading matrices $\mathbf{P}_{i}$ and $\mathbf{M}_{i}$ is not analytically tractable. So we shall consider special cases of the most general problem. In all the cases we assume that the transmitter and receiver are aware of CSI.

\section{A. Only Time Domain Spreading}

In this case $n_{f}=1$. The matrix channel of Equation (23) with output matrix $\mathbf{Y}$ reduces to a vector channel, with vector output $\mathbf{y}$. The transmission model thus becomes

$$
\mathbf{y}=\sum_{i=1}^{N} \sqrt{h_{i} p_{i}} \mathbf{m}_{i} b_{i}+\mathbf{z}
$$

This problem has been solved in [4]. The optimal policy is to allow only those users whose normalized channel gains are above a threshold to transmit in orthogonal channels. The number of such users can't exceed either $n_{t}$, the length of the spreading code in time or $N$, the total number of users in the system. The problem then reduces to independent single user transmissions of [13] for which the optimal solution for each user is to waterfill over the channel fading distribution.

\section{B. Only Frequency Domain Spreading}

In this case there is no CDMA spreading $\left(n_{t}=1\right)$ and the users transmit their information along the $n_{f}$ subcarriers. The matrix channel of Equation (23) with output matrix $\mathbf{Y}$ again reduces to a vector channel, with vector output $\mathbf{y}$ and the transmission model becomes

$$
\mathbf{y}=\sum_{i=1}^{N} \mathbf{H}_{i}^{\frac{1}{2}} \mathbf{p}_{i}^{\frac{1}{2}} b_{i}+\mathbf{z}
$$

where $\mathbf{p}_{i}^{\frac{1}{2}}=\left[\sqrt{p_{i 1}}, \sqrt{p_{i 2}}, \cdots, \sqrt{p_{i n_{f}}}\right]$, the power vector of the $i^{\text {th }}$ user. The corresponding maximum mutual information (MMI), for given channel state matrices for all the users, can be expressed as

$$
\mathbf{R}\left(\mathbf{h}_{1}, \cdots, \mathbf{h}_{N}\right)=\log \left|\mathbf{I}+\sum_{i=1}^{N} \mathbf{H}_{i}^{\frac{1}{2}} \mathbf{p}_{i}^{\frac{1}{2}}\left(\mathbf{h}_{i}\right) \mathbf{p}_{i}^{\frac{1}{2}}\left(\mathbf{h}_{i}\right)^{T}\left(\mathbf{H}_{i}^{\frac{1}{2}}\right)^{T}\right|,
$$

where $|\mathbf{X}|$ denotes det $\mathbf{X}$.

For subsequent rate analysis we do not consider ergodic capacity maximization. This is because we observed in the single user case that ergodic capacity maximization policies involves averaging over the distribution of channel states, which leads to long delays. We expect higher delays in the multi-user case. To avoid long delays, we fix power $\bar{P}$, to be allocated over the subcarriers for each 
channel state realization $\mathbf{h}_{i}$ for all the $i$ users. Thus the optimization of MMI for a given channel realization can be stated as,

$$
\begin{aligned}
\mathrm{R}_{\text {SUM-CSI }} & =\max _{\mathbf{p}_{1}, \cdots, \mathbf{p}_{N}} \mathrm{R}\left(\mathbf{h}_{1}, \cdots, \mathbf{h}_{N}\right) \\
\mathbf{p}_{i}^{\frac{1}{2}}\left(\mathbf{h}_{i}\right)^{T} \mathbf{p}_{i}^{\frac{1}{2}}\left(\mathbf{h}_{i}\right) & =\bar{P} \text { for all } i \\
p_{i j}\left(\mathbf{h}_{i}\right) & \geq 0, \quad 1 \leq i \leq N, 1 \leq j \leq n_{f} .
\end{aligned}
$$

However this problem is non-convex in the power vectors $\mathbf{p}_{i}\left(\mathbf{h}_{i}\right)$ and an algorithmic solution doesn't exist, unlike other multi-user vector transmission problems in which the rate objective is a convex function of the transmit covariance matrices [17]. This non-convexity, stated in a slightly different form, is also observed in [11]. The reason is that transmitted signal $\mathbf{x}_{i}=\sqrt{\mathbf{\mathbf { p } _ { i }}} b_{i}$ lies in an onedimensional space $\left(\operatorname{rank}\left(E\left[\mathbf{x}_{i} \mathbf{x}_{i}^{T}\right]\right)=1\right)$ and it can't waterfill over all the other dimensions, which is the solution for the convex problems of [17].

\section{Proposed Heuristic Solution}

Since the optimal scheme is unknown, we propose a heuristic solution, which is inspired from the iterative water-filling algorithm of [17]. In this heuristic, each user treats the signal of all other user's as noise and chooses the optimal power vectors, in an iterative way. For simplified analysis we consider the problem of only frequency domain spreading as in Section V-B. The proposed heuristic is thus,

1) Denote $\mathbf{x}_{j} \equiv \mathbf{p}_{j}^{\frac{1}{2}}\left(\mathbf{h}_{j}\right)$ for all $j$ Initialize $\mathbf{x}_{j}=\mathbf{x}_{0}$.

2) Repeat

$$
\begin{aligned}
& \text { for } i=1 \text { to } N, j \neq i, \\
& S_{z z i}=\mathbf{I}+\sum_{j} \mathbf{H}_{j}^{\frac{1}{2}} \mathbf{x}_{j} \mathbf{x}_{j}^{T}\left(\mathbf{H}_{j}^{\frac{1}{2}}\right)^{T}, \\
& \mathbf{x}_{i}=\arg \max _{\mathbf{x}_{i}} \log \left|S_{z z i}+\mathbf{H}_{i}^{\frac{1}{2}} \mathbf{x}_{i} \mathbf{x}_{i}^{T}\left(\mathbf{H}_{i}^{\frac{1}{2}}\right)^{T}\right|, \\
& \text { end }
\end{aligned}
$$

until the MMI converges.

It can be shown that the maximization problem in the above algorithm, can be reduced to,

$$
\begin{aligned}
& \max _{\mathbf{x}} \mathbf{x}^{T}\left(\mathbf{H}^{\frac{1}{2}}\right)^{T} \mathbf{S}_{\mathbf{z z}}{ }^{-1} \mathbf{H}^{\frac{1}{2}} \mathbf{x} \\
& \text { s.t. } \quad \mathbf{x}^{T} \mathbf{x}=\bar{X}, \quad \mathbf{x}>0
\end{aligned}
$$

where the user index $i$ has been dropped for simplicity. The solution is outlined in Appendix I. It is shown that the optimal $\mathbf{x}$ lies in the space of the eigenvectors of all the principal sub-matrices of $\mathbf{A}=\left(\mathbf{H}^{\frac{1}{2}}\right)^{T} \mathbf{S}_{\mathbf{z z}}{ }^{-1} \mathbf{H}^{\frac{1}{2}}$, with zeros padded to these eigenvectors to produce the vector $\mathrm{x}$ of length $n$.

Such a solution can be easily implemented in a distributed fashion. The receiver, who has access to all the received signals, can broadcast the total spec$\operatorname{trum} S_{z z}^{(t o t)}=\mathbf{I}+\sum \mathbf{H}_{j}^{\frac{1}{2}} \mathbf{p}_{j}^{\frac{1}{2}}\left(\mathbf{h}_{j}\right) \mathbf{p}_{j}^{\frac{1}{2}}\left(\mathbf{h}_{j}\right)^{T}\left(\mathbf{H}_{j}^{\frac{1}{2}}\right)^{T}$ where the summation is over all the users and user $i$, can subtract his own spectrum from $S_{z z}^{(t o t)}$ to obtain $S_{z z i}$ of the iterative algorithm. Such methods have been discussed in [11]

\section{CONCLUSION}

This paper defines spreading along time and frequency in a generalized form and provides a comprehensive information theoretic analysis for the same. It develops the transmission model for single user, and studies optimal power allocation and transmission schemes, under three different cases of channel state knowledge at the transmitter. For perfect CSI at transmitter the optimal single user policy is to transmit in the best subcarrier and the transmit power is obtained by waterfilling over the distribution of the best subcarrier. For no CSI, the optimal policy is equal power allocation in all subcarriers. For one bit per subcarrier feedback the optimal policy turned out to be to transmit in all the subcarriers that are above the threshold. The work also investigated the asynchronous multi-user transmission and showed that most problems are still open and hence proposed a heuristic solution, which is implementable in a distributed way.

There are several directions of future research. The outage behavior and delay aspects of the proposed model have to be carefully investigated. On a more practical note, since the transmission spans both time and frequency dimensions, scheduling between various transmissions has to be studied, in order to implement the best subcarrier policies for any CSI. In conclusion we note that the multi-user asynchronous problem, can also be addressed by starting with a different objective other than sum capacity. There has been recent works [9], where the multi-user scenario has been modeled as a non cooperative power control game, with the competitive optimality of the users being the objective. These approaches may lead to meaningful models for the physical situation which are at the same time are analytically tractable.

\section{APPENDIX I}

MAximization of $\mathbf{x}^{T} \mathbf{A} \mathbf{x}$ WiTH $\mathbf{x}^{T} \mathbf{x}=1$ AND $\mathbf{x} \geq 0$

The optimization problem, we seek to solve is

$$
\max _{\substack{\mathbf{x}^{T} \mathbf{x}=1 \\ \mathbf{x} \geq 0}} \mathbf{x}^{T} \mathbf{A x},
$$

where $\mathbf{A} \in \mathcal{R}^{n \times n}$ and $\mathbf{x} \in \mathcal{R}^{n}$. To solve the problem for any arbitrary $\mathbf{A}$, we write its Lagrangian as,

$$
\begin{aligned}
& \mathcal{L}=\mathbf{x}^{T} \mathbf{A} \mathbf{x}+\lambda \mathbf{x}^{T} \mathbf{x}+\boldsymbol{\mu}^{T} \mathbf{x}, \\
& \boldsymbol{\mu} \geq 0 \quad \lambda \text { is unconstrained, }
\end{aligned}
$$

where $\lambda$ is a scalar and $\boldsymbol{\mu} \in \mathcal{R}^{n}$. Taking the partial derivative w.r.t. $\mathbf{x}$ yields,

$$
\begin{aligned}
\frac{\partial \mathcal{L}}{\partial \mathbf{x}} & =2 \mathbf{A} \mathbf{x}+2 \lambda \mathbf{x}+\boldsymbol{\mu}=0, \\
\mu_{i} x_{i} & =0,1 \leq i \leq n, \\
\mathbf{x}^{T} \mathbf{x} & =1, \quad \mathbf{x} \geq 0, \boldsymbol{\mu} \geq 0 .
\end{aligned}
$$


The number of zero components of the optimal $\mathbf{x}$ can be from 0 to $n-1$. Let all vectors having $k$ zeros be said to belong to the $k^{\text {th }}$ class. To solve, we consider all the classes one by one and for each class, we solve Equation (35) for $\mathrm{x}$. We retain the vectors those satisfy the original constraints and call them feasible. Ultimately we check which feasible $\mathrm{x}$ maximizes the objective. These are explained in detail below,

1) Class 1: $\mathrm{x}$ has no zero element: In this case the constraints are:

$$
\begin{aligned}
& x_{i}>0,1 \leq i \leq n \\
& \mu_{i}=0,1 \leq i \leq n, \text { from Equation (36) }
\end{aligned}
$$

For these constraints, Equation (35) yields,

$$
\mathbf{A x}=-\lambda \mathbf{x} .
$$

Hence the stationary points of the Lagrangian are given by the unit norm eigenvectors of the original matrix $\mathbf{A}$. The feasible vectors are those, for which constraints in Equation (38) are satisfied.

2) Class 2: $\mathbf{x}$ has one zero element: The zero element can occur in any of the $n$ places. We consider all these sub-classes one by one. Let us begin with $x_{1}=0$. The constraints become

$$
\begin{aligned}
& x_{i}>0,2 \leq i \leq n, \\
& \mu_{1}>0, \\
& x_{1}=0 \text { and } \mu_{i}=0,2 \leq i \leq n .
\end{aligned}
$$

For these parameters, Equation (35) can be expressed as

$$
\mathbf{A x}+\lambda \mathbf{x}=-\frac{1}{2} \boldsymbol{\mu}
$$

This can be rewritten into an eigenvalue problem and a linear equation as follows,

$$
\begin{aligned}
& \mathbf{a}_{1}^{T} \mathbf{x}_{1}=-\frac{1}{2} \mu_{1}, \\
& \mathbf{A}_{1} \mathbf{x}_{1}=-\lambda \mathbf{x}_{1},
\end{aligned}
$$

where,

$$
\begin{aligned}
\mathbf{a}_{1} & =\left[a_{12}, a_{13}, \cdots, a_{1 n}\right], \\
\mathbf{x}_{1} & =\left[x_{2}, x_{3}, \cdots, x_{n}\right], \\
\mathbf{A}_{1} & =\left[\begin{array}{ccc}
a_{22} & \cdots & a_{2 n} \\
\vdots & & \vdots \\
a_{n 2} & \cdots & a_{n n}
\end{array}\right] .
\end{aligned}
$$

Now solve Equation (46) and calculate the unit-norm eigenvectors $\mathbf{x}_{1}$ of $\mathbf{A}_{1}$. Then check if,

1) All elements of $x_{1}$ are positive. [ref. Equation (41)]

2) $\mu_{1}$ calculated from Equation (45) is positive. [ref. Equation (42)]

If $\mathbf{x}_{1}$ satisfies both then form feasible $\mathbf{x}=\left[0, \mathbf{x}_{1}\right]$.

Now consider $x_{k}=0, k \neq 1$. Flip the first and the $k^{t h}$ rows of $\mathbf{A}$ and first and the $k^{\text {th }}$ elements of both $\mathbf{x}$ and $\boldsymbol{\mu}$. The resulting system of equations has the same structure as the $x_{1}=0$ case, and can be solved.
3) Cases $3-n$ : $\mathbf{x}$ has $k$ zero elements, $2 \leq k \leq$ $n$ : The procedure outlined in Section I-.2 can be easily generalized to consider all the remaining cases. We omit a complete proof for sake of brevity. It can be shown that For any $k$, there are $\left(\begin{array}{l}n \\ k\end{array}\right)$ ways in the $k$ zeros can be distributed. in $\mathbf{x}$ and for each sub-class solving Equation (35) reduces to an eigenvalue problem of the matrix $\mathbf{A}_{k} \in \mathcal{R}^{(n-k) \times(n-k)}$ and a system of $k$ linear equations.

Note that this method solves the optimization problem for any arbitrary matrix A. However the complexity of the algorithm increases exponentially with $n$.

\section{REFERENCES}

[1] J. Acharya. Two dimensional spreading for doubly dispersive channels. Master's thesis, WINLAB,Rutgers University, May 2005.

[2] E. Biglieri, J. Proakis, and S. Shamai (Shitz). Fading channels: Information-theoretic and communications aspects. IEEE Trans. Info Theory, 44:2619-2692, Oct. 1998.

[3] G. Caire and S. Shamai. On the capacity of some channels with channel state information. IEEE Trans. Info Theory, 46(6):20072019, Sept 1999.

[4] O. Kaya and S. Ulukus. Jointly optimal power and signature sequence allocation for fading cdma. In Proc. IEEE GLOBECOMM 2003, volume 4, pages 1872-1876, Dec 2003.

[5] R. Knopp and P. A. Humblet. Information capacity and power control in single-cell multiuser communications. ICC '95- IEEE, 1995.

[6] K. Liu, T. Kadous, and A. M. Sayeed. Orthogonal time-frequency signaling over doubly dispersive channels. IEEE Trans. Info Theory, 50(11):2583-2603, Nov 2004.

[7] N. Maeda, H. Atarashi, S. Abeta, and M. Sawahashi. Throughput comparison between VSF-OFCDM and OFDM considering effect of sectorization in forward link broadband packet wireless access. IEEE Trans. on Vehic. Tech., 1, September 2002.

[8] M. Mecking. Resource allocation for fading multiple-access channels with partial channel state information. IEEE International Conf. on Comm., 3:1419 - 1423, April - May 2002.

[9] F. Meshkati, M. Chiang, S. C. Schwartz, H. V. Poor, and N. B. Mandayam. A non-cooperative power control game for multicarrier CDMA systems. Proc. of IEEE WCNC, 2005.

[10] L. H. Ozarow, S. Shamai, and A. D. Wyner. Information theoretic considerations for cellular mobile radio. IEEE Trans. on Vehic. Tech., 43(2):1527 - 1539, May 1994.

[11] D. Popescu and C. Rose. Interference Aviodance Methods for Wireless Systems. Kluwer Academic Publishers.

[12] E. A. Sourour and M. Nakagawa. Performance of orthogonal multicarrier CDMA in a multipath fading channel. IEEE Trans. Commun., 44:356-367, March 1996.

[13] P. P. Varaiya and A. J. Goldsmith. Capacity of fading channels with channel side information. IEEE Trans. Info Theory, 43(6): 1986 - 1992, Nov 1997.

[14] S. Verdu. Multiuser Detection. Cambridge University Press, Cambridge, UK, 1998.

[15] P. Viswanath and V. Anantharam. Optimal sequences and sum capacity of synchronous CDMA systems. IEEE Trans. Info Theory, 45(6):1984-1991, September 1999.

[16] N. Yee, J. Linnartz, and G. Fettweis. Multi-carrier CDMA in indoor wireless radio networks. PIMRC '93, Yokohama, Japan, 1993.

[17] W. Yu, W. Rhee, S. Boyd, and J.M. Cioffi. Iterative water-filling for gaussian vector multiple-access channels. IEEE Trans. Info Theory, 50:145-152, January 2004. 